\title{
Влияние условий формирования кремниевых диодов на их обратные токи
}

\author{
(C) С.В. Булярский ${ }^{1,2}$, Е.П. Кицюк ${ }^{2}$, А.В. Лакалин ${ }^{1,2}$, М.А. Сауров ${ }^{2}$, \\ В.В. Светухин ${ }^{2}$, А.П. Орлов ${ }^{1}$, Г.А. Рудаков ${ }^{1}$ \\ ${ }^{1}$ Институт нанотехнологий микроэлектроники Российской академии наук, \\ 119991 Москва, Россия \\ 2 Научно-производственный комплекс „Технологический центр“, \\ 124498 Москва, Зеленоград, Россия \\ E-mail: bulyar2954@mail.ru
}

Поступила в Редакцию 24 января 2022 г.

В окончательной редакции 31 января 2022 г.

Принята к публикации 31 января 2022 г.

\begin{abstract}
Проведено исследование влияния технологии изготовления кремниевых диодов на возникновение центров генерации и рекомбинации. Сравнивались электрические характеристики $p-n$-переходов, сформированных разными способами на кремниевых подложках $n$-типа: a) слой $p$-типа был создан диффузионным методом; $b)$ слой $p$-типа формировался путем ионной имплантации в предварительно наращенный на подложке эпитаксиальный $n$-слой; $c$ ) на подложке последовательно осаждались два эпитаксиальных слоя $n$ - и $p$-типа. Установлено, что у диодов на основе двойного эпитаксиального слоя прямые и обратные вольт-амперные характеристики обусловлены диффузионным механизмом, а сами структуры имеют низкую концентрацию рекомбинационных центров. В то же время у диодов на основе диффузионного метода и ионной имплантации вольт-амперные характеристики обусловлены генерационно-рекомбинационным механизмом. При обратном смещении существенную роль в формировании вольт-амперных характеристик играют электрон-фононные процессы, а при прямом - рекомбинация носителей в области пространственного заряда $p-n$-перехода. Определены концентрации и энергии центров рекомбинации.
\end{abstract}

Ключевые слова: прямые и обратные вольт-амперные характеристики, $p-n$-переход, диффузия, ионная имплантация, эпитаксия, центры рекомбинации, эффект Пула-Френкеля, электрон-фононное взаимодействие.

DOI: $10.21883 /$ FTP.2022.05.52354.9807

\section{1. Введение}

Величина тока через $p-n$-переход при обратном смещении в большинстве случаев определяется дефектами, которые образуют центры генерации-рекомбинации в области пространственного заряда. В этой области происходят процессы генерации электронов и дырок с участием глубоких уровней [1]. Генерация сопровождается эффектом Пула-Френкеля, также на этот процесс оказывает влияние электрон-фононное взаимодействие, что приводит к плавному росту обратного тока с ростом напряжения [2-4]. С точки зрения диффузионной теории протекания тока $p-n$-перехода обратный ток не должен зависеть от приложенного напряжения [5]. Энергия активации температурной зависимости диффузионного тока должна быть равной энергии ширины запрещенной зоны полупроводника, однако экспериментальные работы показывают, что это не так, и энергия активации меньше ширины запрещенной зоны [2,6]. Это связано с тем, что в большинстве случаев обратный ток является суммой диффузионного тока и тока генерации [7], причем последний преобладает [1-4]. Механизм протекания тока можно определить, изучив его зависимость от температуры. Если экспериментальное значение энергии активации температурной зависимости тока меньше энергии ширины запрещенной зоны, но больше ее половины, то обратный ток определяется процессом генерации электронов и дырок через уровни глубоких центров и не должен зависеть от напряжения смещения на $p-n$-переходе. Генерация электронов и дырок происходит в области пространственного заряда $p-n$-перехода, где существует достаточно сильное электрическое поле, которое оказывает влияние на величину тока. В большинстве работ, которые исследуют обратные вольт-амперные характеристики (BAX) полупроводниковых приборов, это явление остается без внимания, а в других — влияние приложенного напряжения объясняется эффектом Пула-Френкеля $[8,9]$. Коэффициент Пула-Френкеля имеет четко определенное теоретическое значение. Это значение должно быть рассчитано и сопоставлено с его экспериментальной величиной для того, чтобы доказать существование данного эффекта. Однако коэффициент Пула-Френкеля, вычисленный из экспериментальных результатов, может быть больше теоретического, что связано с влиянием электронфононного взаимодействия [2-4,10]. Электрон-фононное взаимодействие может быть значительным тогда, когда центры рекомбинации являются комплексами нескольких точечных дефектов. Состав комплексов зависит от технологии получения и содержания сопутствующих примесей. Высокая температура синтеза приводит к повышенной концентрации вакансий [11]. Вакансии в 
кремнии легко захватываются кислородом и образуют комплексы вакансия-кислород. Такие комплексы могут содержать различное число как вакансий, так и атомов кислорода [12]. Они создают в запрещенной зоне кремния центры рекомбинации вблизи середины запрещенной зоны и активно оказывают влияние на величину обратных токов $[2-4,13,14]$. Если влияние кислорода на величину обратных токов $p-n$-переходов исследовано достаточно хорошо, то влияние азота изучено недостаточно, а он оказывает влияние как на образование кислородных комплексов [15], так и сам создает глубокие уровни в запрещенной зоне кремния [12]. Цель данной работы заключается в изучении влияния условий формирования диодов на вольт-амперные характеристики $p-n$-переходов. Для реализации этой цели сравниваются электрические свойства $p-n$-переходов, полученных на монокристаллических подложках кремния, в том числе с эпитаксиальными слоями кремния, отличающимися температурой процесса эпитаксии и составом газовой фазы в реакторе.

\section{2. Экспериментальные результаты}

Для экспериментов были подготовлены пластины кремния тремя различными способами. Объекты группы „А“ были созданы на пластинах низколегированного кремния $n$-типа, полученных методом бестигельной зонной плавки. Удельное сопротивление подложки образцов „А“ составляло $\sim 4400$ Ом · см. Слой $p$-типа толщиной 0.5 мкм с концентрацией легирующей примеси $10^{19} \mathrm{~cm}^{-3}$ был создан методом ионной имплантации примеси бора. Диоды группы „В“ формировались на низкоомных пластинах кремния $n$-типа. На них последовательно наращивался эпитаксиальный слой $n$-типа толщиной 30 мкм при температуре $1050^{\circ} \mathrm{C}$ методом разложения моносилана. Реактор продувался азотом перед синтезом образцов группы „В“. Далее методом ионной имплантации формировался $p^{+}$-слой толщиной 0.5 мкм с концентрацией $10^{20} \mathrm{~cm}^{-3}$. Концентрация бора и азота в эпитаксиальных слоях диодов группы „В“ измерялась методом времяпролетной вторичноионной масс-спектрометрии на спектрометре ToFSIMS-5 фирмы IonTOF GmbH. Концентрация бора составила $2 \cdot 10^{18} \mathrm{~cm}^{-3}$, а азота $-10^{21} \mathrm{~cm}^{-3}$. По-видимому, использование азота для продувки реактора перед эпитаксиальным осаждением способствовало его накоплению в $p^{+}$-слое диодов „В“. Пластины группы „С“ получали на пластинах низкоомного кремния последовательным осаждением двух эпитаксиальных слоев $n$ - и $p$-типа при температуре $950^{\circ} \mathrm{C}$ из трихлорсилана и водорода. Все технологические процессы проводились с использованием аргона и водорода высокой степени очистки без следов примесей кислорода и азота. Концентрация кислорода в исходных пластинах группы „С“ контролировалась по спектрам инфракрасного поглощения и составляла $8 \cdot 10^{17} \mathrm{~cm}^{-3}$.
Все диоды после их изготовления были $p^{+}-n$-типа, имели $p^{+}$-область с высоким уровнем легирования и обладали резким $p-n$-переходом. Для измерения вольтамперных и вольт-фарадных характеристик этих диодов использовался автоматизированный комплекс на основе анализатора полупроводниковых приборов Keysight B1500A. Концентрация свободных носителей заряда в низколегированной области $n$-типа вычислялась по результатам измерения вольт-фарадных характеристик. Она составляла для образцов различных групп: „А“ $(5.9 \pm 0.2) \cdot 10^{11} \mathrm{~cm}^{-3} ; \quad{ }_{\text {}} \mathrm{B}^{6 /}-(3.0 \pm 0.5) \cdot 10^{16} \mathrm{~cm}^{-3}$; „C' $-(2.2 \pm 0.2) \cdot 10^{14} \mathrm{~cm}^{-3}$.

Обратные вольт-амперные характеристики диодов представлены на рис. 1.

\section{3. Обсуждение результатов}

Энергия активации температурной зависимости обратного тока зависит от основного механизма, который формирует этот ток. Энергия активации, близкая к ширине запрещенной зоны $\left(E_{g}\right)$, соответствует диффузионному механизму. Энергия активации, которая лежит в диапазоне $0.5 \cdot E_{g}<E_{a}<E_{g}$, соответствует генерации через центры рекомбинации в запрещенной зоне области полупроводника, которая легирована меньше. Энергии активации были вычислены с использованием координат Аррениуса по данным, приведенным на рис. 1, и при различных напряженностях электрического поля. Для построения зависимости было выбрано максимальное значение электрического поля в области пространственного заряда диодов, которое равно удвоенному среднему значению поля, так как понижение энергии активации происходит именно на границе, где поле максимально. Результаты вычислений представлены на рис. 2.

Энергия активации обратного тока в диодах группы „С“ приближается к энергии ширины запрещенной зоны в кремнии. Это указывает, что механизм протекания обратного тока в них является диффузионным, и обратный диффузионный ток превышает ток генерации через локальные электронные состояния глубоких уровней. Это не означает, что дефекты не оказывают влияния на величину обратного тока диодов „С“. Содержание глубоких уровней определяет время жизни носителей заряда в соответствующих областях. Это время можно вычислить из величины тока насыщения [5]. Рекомбинация происходит в квазинейтральной $n$-области, где неосновными носителями являются дырки, тогда

$$
\tau_{p}=D_{p}\left(\frac{q n_{i}^{2} S}{n_{n} I_{s}}\right)^{2},
$$

где $D_{p}-$ коэффициент диффузии дырок в кремнии, $q$ - заряд электрона, $n_{i}$ - концентрация собственных носителей заряда в кремнии, $S$ - площадь диода, $n_{n}-$ концентрация собственных носителей заряда в $n$-области кремния, $I_{s}$ - ток насыщения диода. 

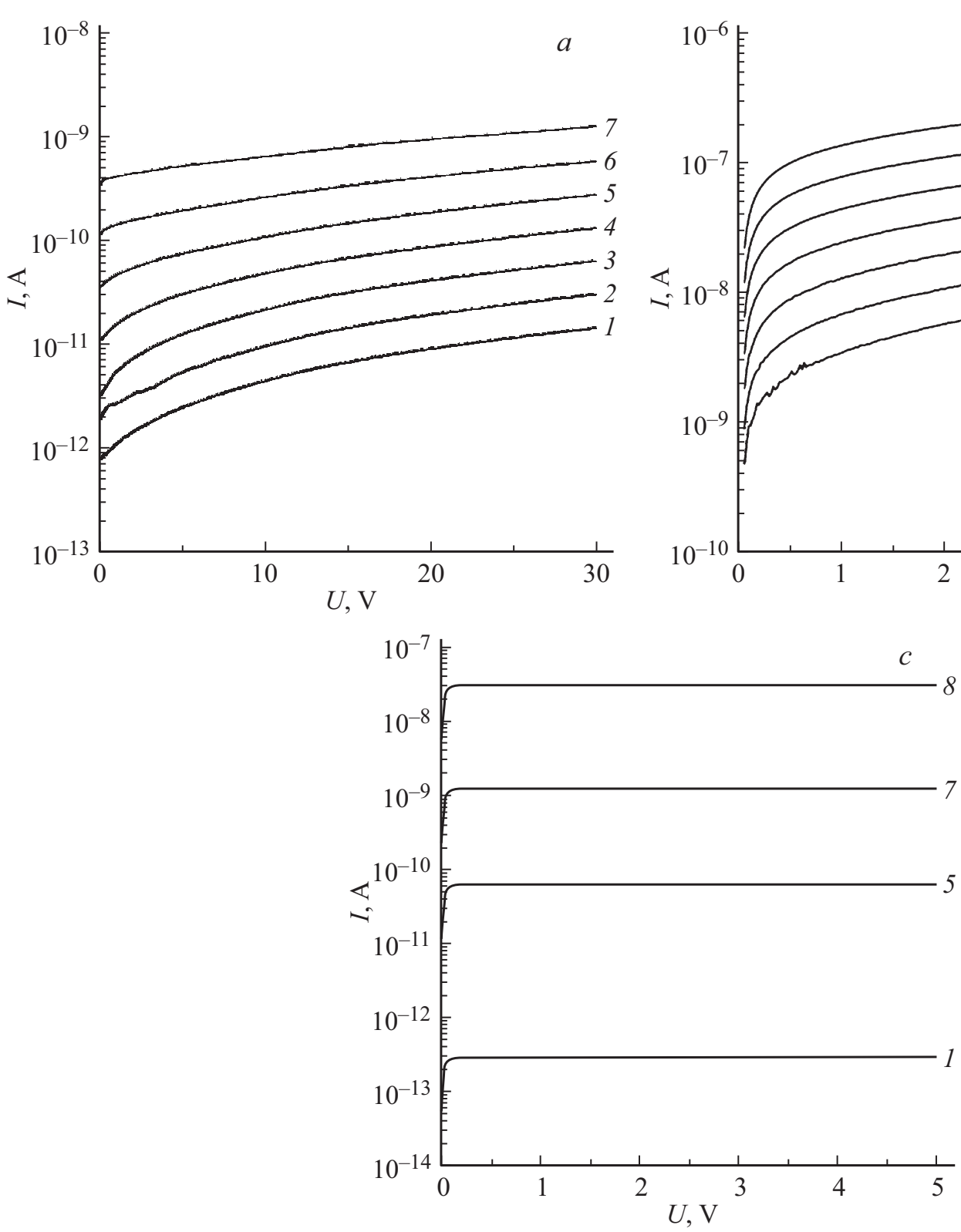

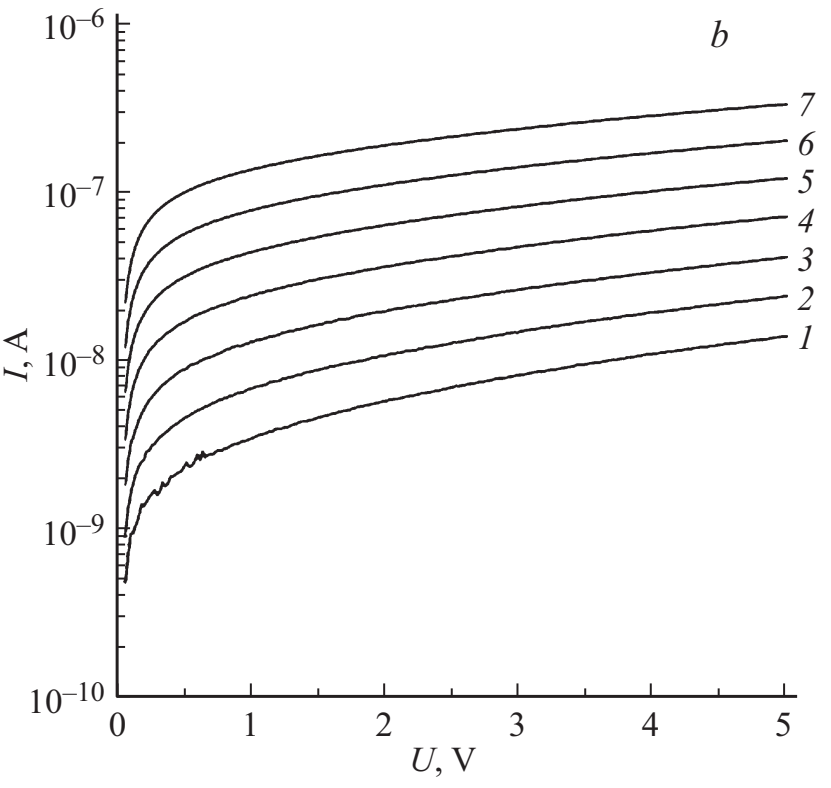
8

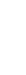

Рис. 1. Обратные вольт-амперные характеристики образцов групп „А“ $(a)$, „В“ $(b)$, „С“ $(c)$, измеренные при температурах, ${ }^{\circ} \mathrm{C}$ : $1-0,2-10,3-20,4-30,5-40,6-50,7-60,8-80$.

Оценка времени жизни по формуле (1) дает для диодов группы „С“ величину $3 \cdot 10^{-4} \mathrm{c}$. Величина времени жизни дырок достаточно высока, что говорит о малой концентрации рекомбинационных центров в области пространственного заряда этих диодов. Действительно, емкостное измерение концентрации глубоких центров этих диодов дали значения концентрации $5 \cdot 10^{10} \mathrm{~cm}^{-3}$ и энергии активации 0.48 эВ. Величина времени жизни и концентрации глубоких центров говорят о высоком качестве двойных эпитаксиальных диодов группы „С“.

Как было отмечено выше, при наличии эффекта Пула-Френкеля энергия активации обратного тока зависит от электрического поля, что связано с понижением высоты потенциального барьера вблизи глубокого центра в электрическом поле:

$$
\Delta E_{t}=\frac{q^{3 / 2}}{\sqrt{\pi \varepsilon_{S}}} \sqrt{F}=\beta_{\mathrm{FT}} \sqrt{F},
$$

где $\varepsilon_{S}$ - статическая диэлектрическая проницаемость полупроводника; $\beta_{\mathrm{FT}}-$ теоретическое значение константы Пула-Френкеля, которое для кремния равно 0.00023 эВ $\cdot \mathrm{cm}^{1 / 2} / \mathrm{B}^{1 / 2}$.

Для того чтобы найти энергии активации центров рекомбинации, проводилась процедура экстраполяции методом регрессии. Кроме того, принималось во внимание, что ток генерации ограничивается электронным переходом, который имеет большую энергию актива- 


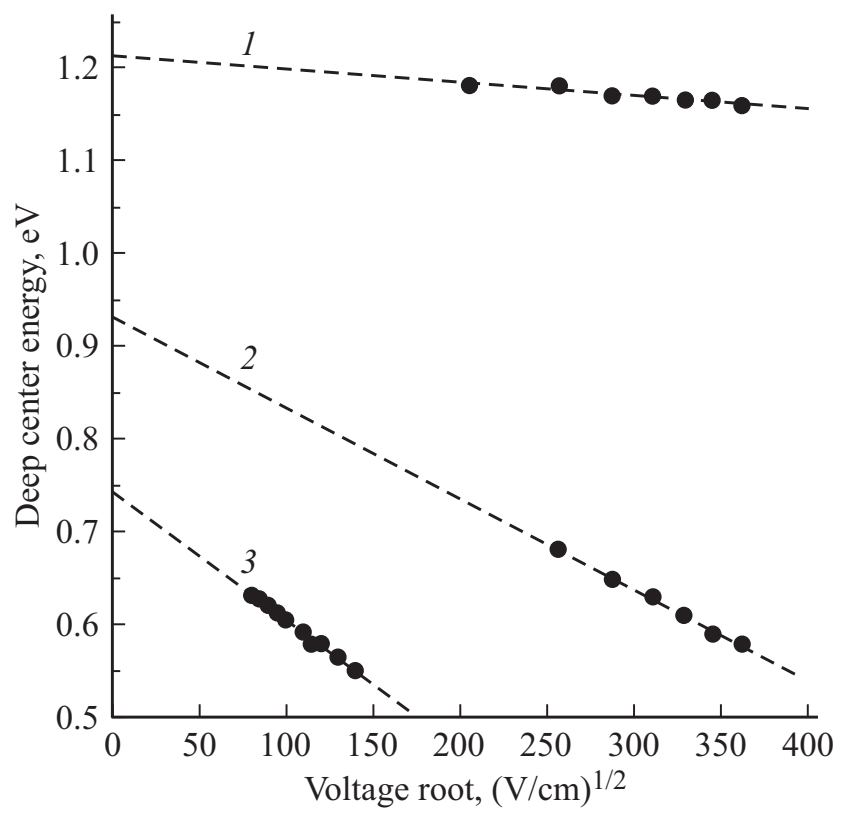

Рис. 2. Зависимость энергии активации обратного тока от величины максимальной напряженности поля в области пространственного заряда диодов: 1 - „С“, 2 - „В“, 3 - „А“.

ции [2-4]. Чтобы найти энергию активации центра, через который идет генерация, необходимо от ширины запрещенной зоны кремния при $0 \mathrm{~K}$ вычесть значение энергии, которое получено экстраполяцией к нулевой напряженности электрического поля. Для диодов группы „А“ получается энергия 0.47 эВ и константа Пула-Френкеля $(1.34 \pm 0.03) \cdot 10^{-3}{ }_{э} \mathrm{~B} \cdot \mathrm{cm}^{1 / 2} / \mathrm{B}^{1 / 2}$, а для диодов группы „В“ получается энергия 0.29 эВ и константа $(1.02 \pm 0.03) \cdot 10^{-3}$ эВ $\cdot \mathrm{cm}^{1 / 2} / \mathrm{B}^{1 / 2}$. В обоих случаях константа Пула-Френкеля превышает теоретическое значение, что связано с электрон-фононным взаимодействием. В работе С.Ф. Тимашева [16] показано, что

$$
\beta=\beta_{\mathrm{FT}}\left(1+\frac{E_{0}-S \hbar \omega}{S \hbar \omega}\right),
$$

где $S \hbar \omega$ - величина, равная половине тепловыделения, которое сопровождает электрон-фононное взаимодействие; $E_{0}$ - энергия чисто электронного перехода.

Выражение (3) справедливо, когда генерация носителей заряда происходит в результате многофононного процесса, и в нем принимает участие только один тип фононов, имеющих некоторую эффективную энергию. Алгоритм вычисления параметров электрон-фононного взаимодействия изложен в работах [2-4]. Анализ публикаций по дефектам в кремнии показывает, что энергия активации $0.47-0.48$ эВ может соответствовать комплексу $\mathrm{V}_{\mathrm{Si}}-\mathrm{O}[12,16-18]$ либо дивакансии $[12,13,19]$. Центр рекомбинации 0.25 эВ связан с внедрением азота в решетку кремния [16]. Таким образом, центры рекомбинации в диодах групп „А“ и „В“ обусловлены комплексами кислорода с вакансией кремния. В диодах, изготовленных методом последовательной двойной эпитаксии „С“, центров рекомбинации существенно меньше, и они не определяют обратный ток данных диодов.

В ряде работ, в том числе работах [1,2], применяется метод рекомбинационной спектроскопии, который заключается в преобразовании прямых вольт-амперных характеристик по определенным алгоритмам для появления на первоначально монотонной вольт-амперной характеристике некоторых особенностей. Эти особенности позволяют оценить параметры центров рекомбинации.
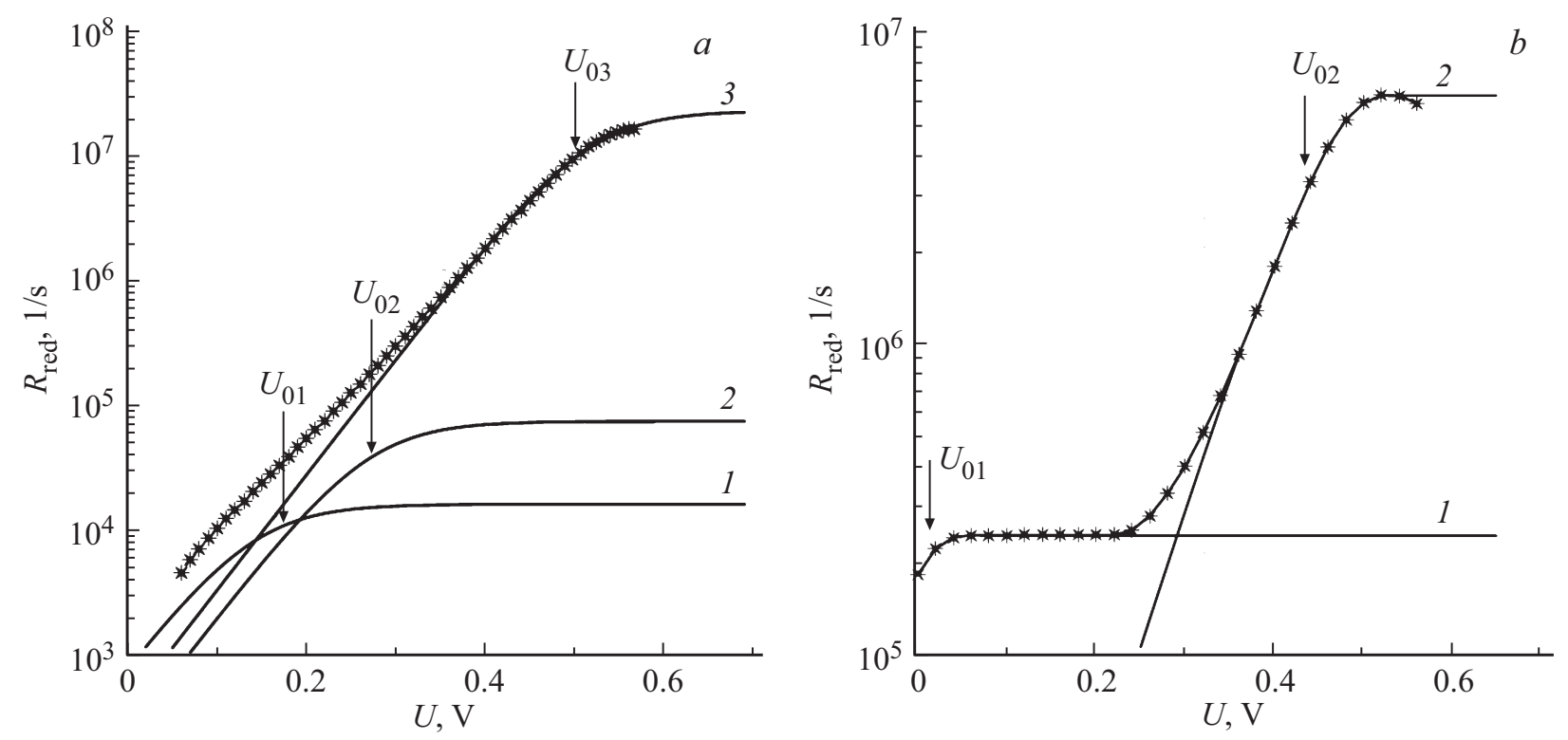

Рис. 3. Приведенная скорость рекомбинации диодов групп „А“ $(a)$ и „В“ $(b)$, вычисленная при температуре $0^{\circ} \mathrm{C}$. Стрелками отмечены напряжения, при которых приведенная скорость рекомбинации насыщения уменьшается в 2 раза. Цифры отмечают номер рекомбинационного процесса. 
Один из таких алгоритмов заключается в вычислении величины приведенной скорости рекомбинации $\left(R_{\mathrm{red}}(U)\right)$, которая имеет смысл обратного времени жизни. Эта величина вычисляется из экспериментальной вольтамперной характеристики по формуле

$$
R_{\mathrm{red}}(U)=\frac{I_{r}(U)}{q S w(U) n_{i}\left[\exp \left(\frac{q U}{2 k T}\right)-1\right]} \frac{q\left(V_{d}-U\right)}{2 k T},
$$

где $U$ - текущее значение напряжения прямого смещения, $I_{r}(U)$ - текущее значение тока рекомбинации в области пространственного заряда, $w(U)$ - ширина области пространственного заряда, $V_{d}$ - диффузионный потенциал $p-n$-перехода.

Приведенные скорости рекомбинации диодов групп „A“ и „В“ при температуре $0^{\circ} \mathrm{C}$ показаны на рис. 3 .

Энергию активации рекомбинационных центров можно оценить с помощью простой формулы [1,2]

$$
E_{t}=\frac{E_{g}-q U_{0}}{2}+\delta
$$

где

$$
\delta=\frac{k T}{2} \ln \left(\frac{1}{4} \frac{c_{n} N_{c}}{c_{p} N_{v}}\right)
$$

Здесь $U_{0}$ - напряжение, при котором приведенная скорость рекомбинации насыщения уменьшается в 2 раза, $E_{g}$ - ширина запрещенной зоны кремния при комнатной температуре, $c_{n}$ - коэффициент захвата электронов на центр рекомбинации, $c_{p}-$ коэффициент захвата дырок на центр рекомбинации, $N_{v}$ - эффективная плотность электронных состояний валентной зоны, $N_{c}-$ эффективная плотность электронных состояний зоны проводимости.

Коэффициенты захвата нам не известны, тем не менее формула (5) позволяет оценить энергию активации с точностью до 0.02 эВ. Приведенная скорость рекомбинации насыщения равна $R_{\text {red }}^{\max }=\sqrt{c_{n} c_{p}} N_{t} / 2$.

Ее обратная величина позволяет сделать оценку времени жизни при рекомбинации через конкретный центр. У образцов группы „А“ таких центров рекомбинации три: $E_{t 1}=0.48, E_{t 2}=0.44, E_{t 3}=0.33$ эВ; времена жизни при рекомбинации через них равны соответственно: $5 \cdot 10^{-5}, 1.3 \cdot 10^{-5}, 5 \cdot 10^{-8}$ с. У образцов группы „В“ два типа центров рекомбинации: $E_{t 1}=0.58, E_{t 2}=0.34$ эВ; времена жизни при рекомбинации через них равны соответственно: $4 \cdot 10^{-6}, 1.6 \cdot 10^{-7}$ с. В целом результаты оценки энергии активации центров рекомбинации по прямым и обратным характеристикам согласуются между собой. Энергия 0.33 эВ имеет центр рекомбинации, который связан с дивакансией в кремнии. Поэтому вопрос о природе данного центра требует дальнейшего изучения. Не исключено, что так как энергии активации центров азота и дивакансии близки, то процессы рекомбинации накладываются друг на друга и данный метод не позволяет разделить эти процессы.

\section{4. Заключение}

Особенности технологии получения $p-n$-переходов кремния приводят к различному набору центров рекомбинации. Создание диодов на кремнии, который выращен методом бестигельной зонной плавки или методом Чохральского, проводится при температурах, близких к температуре плавления кремния, кроме того, эти монокристаллы содержат много кислорода. Поэтому неизбежно наличие центров рекомбинации, которые связаны с комплексами кремния с кислородом. Эти центры снижают время жизни и увеличивают токи утечки. Обратные BAX при наличии таких центров изменяются плавно. Применение азота в технологическом процессе способно создать дополнительные центры рекомбинации. Диоды, полученные методом двойной эпитаксии, имеют мало центров рекомбинации, их ВАХ описываются диффузионным механизмом протекания тока с достаточно большим временем жизни, чтобы обратный ток при комнатной температуре был мал. Это связано с тем, что относительно низкая температура эпитаксии приводит к малой концентрации вакансий, которая с уменьшением температуры синтеза кремния падает экспоненциально. Применение в процессе синтеза особо чистого водорода снижает концентрацию кислорода в эпитаксиальных слоях. Все это вместе взятое ведет к уменьшению концентрации центров рекомбинации.

\section{Благодарности}

Авторы работы выражают благодарность сотрудникам ИНМЭ РАН М.Г. Верхолетову и А.В. Горячеву за помощь в проведении измерений.

\section{Финансирование работы}

Работа выполнена при поддержке Министерства науки и высшего образования Российской Федерации, проект № 0004-2022-0004.

\section{Конфликт интересов}

Авторы заявляют, что у них нет конфликта интересов.

\section{Список литературы}

[1] С.В. Булярский, А.Н. Сауров. Физика полупроводниковых преобразователей (М., РАН, 2018).

[2] S.V. Bulyarskiy. Solid-State Electron., 160 (9), 107624 (2019). https://doi.org/10.1016/j.sse.2019.107624

[3] С.В. Булярский, А.В. Лакалин, М.А. Сауров. ФТП, 55 (1), 69 (2021). https://doi.org/10.21883/FTP.2021.01.50389.9455

[4] S.V. Bulyarskiy, A.V. Lakalin, M.A. Saurov, G.G. Gusarov. J. Appl. Phys., 128 (15), 155702 (2020). https://doi.org/10.1063/5.0023411

[5] W. Shockley. Bell Syst. Techn. J., 28 (3), 435 (1949). https://doi.org/10.1002/j.1538-7305.1949.tb03645.x 
[6] K.A. Abdullah, F.A. Alloush, A. Jaafar, C. Salame. Energy Procedia, 36, 104 (2013). https://doi.org/10.1016/j.egypro.2013.07.013

[7] C.-T. Sah, R. Noyce, W. Shockley. Proc. IRE, $45(9), 1228$ (1957). https://doi.org/10.1109/JRPROC.1957.278528

[8] Q. Shan, D.S. Meyaard, Q. Dai, J. Cho, F. Schubert E., J. Kon Son, C. Sone. Appl. Phys. Lett., 99(25), 253506 (2011). https://doi.org/10.1063/1.3668104

[9] M. Musolino, D. van Treeck, A. Tahraoui, L. Scarparo, C. de Santi, M. Meneghini, E. Zanoni, L. Geelhaar, H. Riechert. J. Appl. Phys., 119 (4), 44502 (2016). https://doi.org/10.1063/1.4940949

[10] С.Ф. Тимашев. ФТТ, 16, 804 (1974).

[11] G. Kissinger, J. Dabrowski, T. Sinno, Y. Yang, D. Kot, A. Sattler. J. Cryst. Growth, 468 (4), 424 (2017). https://doi.org/10.1016/j.jcrysgro.2016.10.073

[12] S. Selberherr, P. Pichler. Intrinsic Point Defects, Impurities, and Their Diffusion in Silicon (Vienna, Springer Vienna, 2004).

[13] J.H. Evans-Freeman, A.R. Peaker, I.D. Hawkins, P.Y.Y. Kan, J. Terry, L. Rubaldo, M. Ahmed, S. Watts, L. Dobaczewski. Mater. Sci. Semicond. Process., 3(4), 237 (2000). https://doi.org/10.1016/S1369-8001(00)00038-X

[14] I. Capan, Ž. Pastuović, R. Siegele, R. Jaćimović. Nucl. Instrum. Meth. Phys. Res. Sect. B, 372 (3), 156 (2016). https://doi.org/10.1016/j.nimb.2015.12.039

[15] Y. Qin, P. Wang, S. Jin, C. Cui, D. Yang, X. Yu. Mater. Sci. Semicond. Process., 98, 65 (2019). https://doi.org/10.1016/j.mssp.2019.03.027

[16] S.D. Brotherton, P. Bradley. J. Appl. Phys., 53 (8), 5720 (1982). https://doi.org/10.1063/1.331460

[17] J.M. Meese, J.W. Farmer, C.D. Lamp. Phys. Rev. Lett., 51 (14), 1286 (1983). https://doi.org/10.1103/PhysRevLett.51.1286

[18] A.M. Frens, M.T. Bennebroek, A. Zakrzewski, J. Schmidt, W.M. Chen, E. Janzén, J.L. Lindström, B. Monemar. Phys. Rev. Lett., 72 (18), 2939 (1994). https://doi.org/10.1103/PhysRevLett.72.2939

[19] G. Alfieri, E.V. Monakhov, B.S. Avset, B.G. Svensson. Phys. Rev. B, 68 (23), 2653 (2003). https://doi.org/10.1103/PhysRevB.68.233202

Редактор Г.А. Оганесян

\section{Influence of formation conditions of silicon diodes on their reverse currents}

\author{
S.V. Bulyarskiy 1,2, E.P. Kitsyuk ${ }^{2}$, A.V. Lakalin ${ }^{\mathbf{1 , 2}}$, \\ M.A. Saurov', V.V. Svetukhin' ${ }^{2}$, A.P. Orlov', \\ G.A. Rudakov ${ }^{1}$ \\ ${ }^{1}$ Institute of Nanotechnology of Microelectronics \\ of the Russian Academy of Sciences, \\ 119991 Moscow, Russia \\ 2 Scientific-Manufacturing Complex \\ "Technological Centre“, \\ 124498 Moscow, Zelenograd, Russia
}

Abstract In this work, a study was made of the influence of silicon diode manufacturing technology on the emergence of generation and recombination centers. The electrical characteristics of $p-n$ junctions formed in different ways on $n$-type silicon substrates were compared: $a$ ) the $p$-type layer was created by the diffusion method; $b$ ) the $p$-type layer was formed by ion implantation into an epitaxial $n$-layer preliminarily grown on the substrate; $c$ ) two $n$ - and $p$-type epitaxial layers were successively deposited on the substrate. It has been established that for diodes based on a double epitaxial layer, the direct and reverse currentvoltage characteristics are due to the diffusion mechanism, and the structures themselves have a low concentration of recombination centers. At the same time, in diodes based on the diffusion method and ion implantation, the current-voltage characteristics are due to the generation-recombination mechanism. With reverse bias, electron-phonon processes play a significant role in the formation of the current-voltage characteristics, and with forward bias, carrier recombination in the region of the space charge of the $p-n$ junction. The concentrations and energies of recombination centers have been determined. 